\title{
"Use Judiciously, or I Will Be Useless" A Clinical Audit on Use of Antibiotics Within First 72 hrs of Life in Symptomatic Term Babies With no Maternal Risk for Infection
}

\author{
Khalil Salameh*, Abedal khalik Ahmad Khedr, Rajesh Pattu Valappil and Ahmed Hosny Ahmed Tomerak
}

Department of Pediatrics, Qatar

*Corresponding author: Khalil Salameh, Department of Pediatrics, Qatar

\section{Background}

Over use of antibiotics lead to resistance and unwanted sideeffects in newborns. Since 1992, professional societies or public health agencies have issued several generations of recommendations for prevention or management of early-onset neonatal sepsis (EOS) [1-5]. Despite these efforts, recommendations remain inconsistent, clarifications are necessary, local adaptations are common, and compliance rates are low [6-7]. It is common clinical practice to discontinue antibiotic treatment of asymptomatic babies if the blood cultures are negative at 48 hours [8-10]. But it is very common to have prolonged antibiotic courses for more than 48 hours due to delayed release of blood culture, high CRP, abnormal CBC or delayed decision by the Physician to stop antibiotics. In a previous study, McDonald et al. [11] found this as a common occurrence in neonatal intensive care units. The purpose of this audit is to rationalize the use of antibiotics in symptomatic term babies, with no risk for infection.

\section{Methodology}

This is a retrospective audit to determine the Use of antibiotics in symptomatic term babies with no maternal risk for infection. Any full-term infant who was started on antibiotics after admitting to NICU with various symptoms were included. Preterm babies and babies with maternal risk for infection (i.e. suspected maternal chorioamnionitis, PROM >18 hrs, GBS bacteriuria in mother) were excluded from the audit. Data were collected from January to March 2017 and analyzed with descriptive statistics. A re-audit was carried out between January to March 2018. 35 patients were included in the first audit and 22 in the second cycle. As a result of the first audit cycle following changes in practice were implemented. a) Infants with mild-to-moderate respiratory findings (flaring, grunting, retractions or tachypnea) immediately after birth may be monitored closely for resolution of transitional behaviors, without initiation of antibiotic treatment unless signs worsen or persist for more than 6 hours without improvement [12].

b) Serial normal diagnostic tests, such as blood counts or C-reactive protein levels, are highly predictive of the absence of infection and should be relied upon (in addition to culture results) to minimize the duration of antibiotic exposure. However, isolated abnormal hematological or acute-phase-reactant measurements should not justify continuation of empirical antibiotics for more than 48 hours in well-appearing infants with negative culture results.

c) Babies diagnosed as TTN without another differential diagnosis, should not be started on antibiotics unless mild to moderate respiratory signs persist beyond 6 hours. The re-audit evaluated improvement in clinical practice regarding judicious use of antibiotics.

\section{Results and Conclusion}

Overall the unit adhered well to the guidelines and there was a significant reduction in use of antibiotics in the unit. None of the babies with mild respiratory distress received Antibiotics before 6 hours in the re-audit, where as a significant number (15 babies $(42 \%)$, with mild respiratory distress that improved in 6 hours received antibiotics during the first audit period. Similarly, in none of the babies antibiotics were continued for more than 48 hours for high CRP. (4 babies initial CRP were high). During the first 
audit period, $11 \%$ of the cases antibiotics were continued beyond 48 hours just for high CRP. But when compared to the first audit, more number of babies received antibiotics for cases diagnosed as TTN because the local and international guidelines suggest to start antibiotics in babies with respiratory distress persisting for more than 6 hours.

\section{References}

1. Committee on Infectious Diseases, Committee on Fetus and Newborn (1992) Guidelines for prevention of group B streptococcal (GBS) infection by chemoprophylaxis. Pediatrics 90(5): 775-778.

2. Committee on Technical Bulletins (1993) Group B streptococcal infections in pregnancy. ACOG Technical Bulletin Number 170. Int J Gynaecol Obstet 42(1): 55-59.

3. Centers for Disease Control and Prevention (1996) Prevention of perinatal group B streptococcal disease: a public health perspective. MMWR Recomm Rep 45(RR-7): 1-24.

4. Committee on Obstetric Practice (1996) Prevention of early-onset group B streptococcal disease in newborns. ACOG Committee Opinion Number 173. Int J Gynaecol Obstet 54: 197-205.
5. Committee on Infectious Diseases, Committee on Fetus and Newborn (1997) Revised guidelines for prevention of early-onset group B streptococcal (GBS) infection. Pediatrics 99(3): 489-496.

6. Brady MT, Polin RA (2013) Prevention and management of infants with suspected or proven neonatal sepsis. Pediatrics 132(1): 166-168.

7. Mukhopadhyay S, Dukhovny D, Mao W, Eichenwald EC, Puopolo KM (2014) 2010 perinatal GBS prevention guideline and resource utilization. Pediatrics 133(2): 196-203.

8. Pichichero ME, Todd JK (1979) Detection of neonatal bacteremia. J Pediatr 94(6): 958-960.

9. Rowley AH, Wald ER (1986) Incubation period necessary to detect bacteremia in neonates. Pediatr Infect Dis J 5(5): 590-591.

10. Hurst MK, Yoder BA (1995) Detection of bacteremia in young infants: is 48 hrs adequate? Pediatr Infect Dis J 14(8): 711-713.

11. McDonald M, Moloney A, Clarke TA, et al. (1992) Blood cultures and antibiotic use in a neonatal intensive care unit. Ir J Med Sci 161: 163-4

12. William E Benitz, James L Wynn, Richard A Polin (2015) Reappraisal of Guidelines for Management of Neonates with Suspected Early-Onset Sepsis. J Pediatr 166(4): 1070-1074.

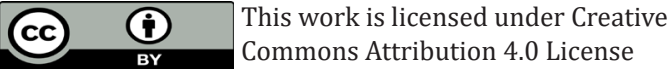

To Submit Your Article Click Here:

Submit Article
DOI: $10.32474 /$ PAPN.2019.02.000128

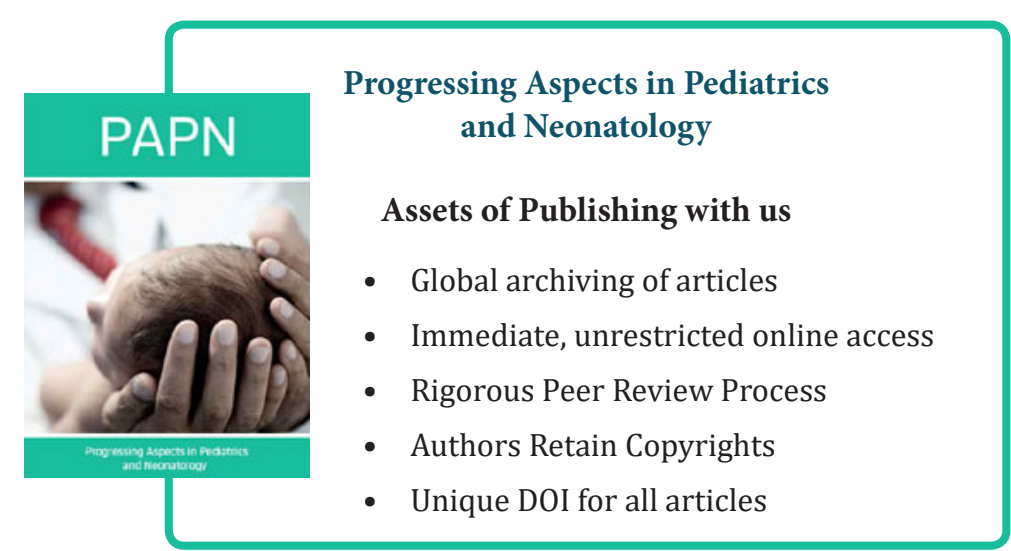

